\title{
Unusual MRI appearance of diffuse subcortical heterotopia or "double cortex" in two children
}

\author{
J H Livingston, J Aicardi
}

\begin{abstract}
Two children with mild epilepsy and learning and behaviour problems had magnetic resonance imaging (MRI) scans showing an almost identical generalised disorder of neuronal migration. Their computer tomography (CT) scans showed abnormal hypodense white matter. The MRI showed a four layered appearance of the cerebral parenchyma extending from the frontal to the occipital region. There was a normal appearance to the white matter in the periventricular region which had an abnormally smooth junction with a thick diffuse layer of heterotopic grey matter. This was surrounded by a thin layer of white matter which had normal digitations with the overlying cortex. The appearance of the overlying cortex was normal. These and other recently described cases broaden the concept of generalised disorders of neuronal migration and illustrate that it is possible to have a generalised cerebral malformation with few clinical consequences.
\end{abstract}

Abnormal neuronal migration occurs in many different neurological diseases and may result in heterotopias of neurons or malformations of the cortical structure. Although these malformations are usually described in neurologically abnormal patients, lesser degrees of heterotopia or cortical abnormalities are not infrequently found at necropsy in the brains of patients who had no or few clinical neurological abnormalities.

When diffuse, disorders of neuronal migration produce large heterotopic masses or abnormalities of gyrus formation, such as agyria, pachygyria or microgyria, usually associated with severe intellectual deficit and often epilepsy. ${ }^{1}$ These diffuse abnormalities can be diagnosed by CT scan. MRI imaging with its greater ability to demonstrate white/ grey differences has greatly facilitated the diagnosis of lesser degrees of pachygyria and agyria. $^{23}$

With refinements in both computer tomography (CT) scanning and magnetic resonance imaging (MRI), focal forms of cortical dysplasia are being increasingly recog- nised. ${ }^{4}$ We report two cases with an almost identical radiological appearance of a diffuse subcortical heterotopia associated with easily controlled epilepsy and mild learning difficulties.

\section{Case report}

Case 1 This was a seven year old girl referred for assessment of seizures. She was the sixth child of non consanguineous parents. There was no family history of note. The pregnancy and birth were uncomplicated with a birth weight of $3.6 \mathrm{kgs}$ at term.

Her early development was slightly slower compared with her siblings. She walked at 18 months, had only simple phrases at three years and subsequently required speech therapy. She had a behaviour problem characterised by episodes of rage and aggression and she was in a special class in a normal school. At the age of six and a half years she began to have seizures, typically in the early mornings on waking, occurring in clusters and lasting up to 20 minutes. These comprised sudden flexion of the trunk with adduction of the arms, upward turning of the eyes and salivation. This would occur once every 10-15 seconds. They usually occurred in bed and would occur three or four times per week. An EEG at that time showed frequent generalised paroxysmal activity with a slight right sided predominance, particularly during hyperventilation. She was not treated at that time and continued to have one to two seizures per month until she was referred at the age of seven years.

Clinical examination was normal apart from slight convergent strabismus. There were no abnormal neurological signs and occipitofrontal circumference was $53.3 \mathrm{cms}$ (plus $1 \mathrm{SD})$. Further EEG at that time showed a slightly slow background with frequent bursts of polyspike/wave at 2.5 cycles per second lasting three to four seconds. Treatment with sodium valproate was started with a good response. CT scan showed moderate dilatation of the lateral ventricles with a large cavum vergae and cyst of the septum pellucidum. There was marked hypodensity of white matter with a very straight white/ grey junction but the cortical appearance was not typical of pachygyria/lissencephaly. 
Figure 1 Case 1: (a) CT scan showing thick cortex and straight white/grey junction. Note enlarged ventricles and cavum septi pellucidi. (b) $T_{2}$ weighted MRI, coronal cut. The thick cortex is divided by a dark line (arrowheads) into a superficial "true" cortex and a deep layer of grey matter separated from white matter by a straight line.
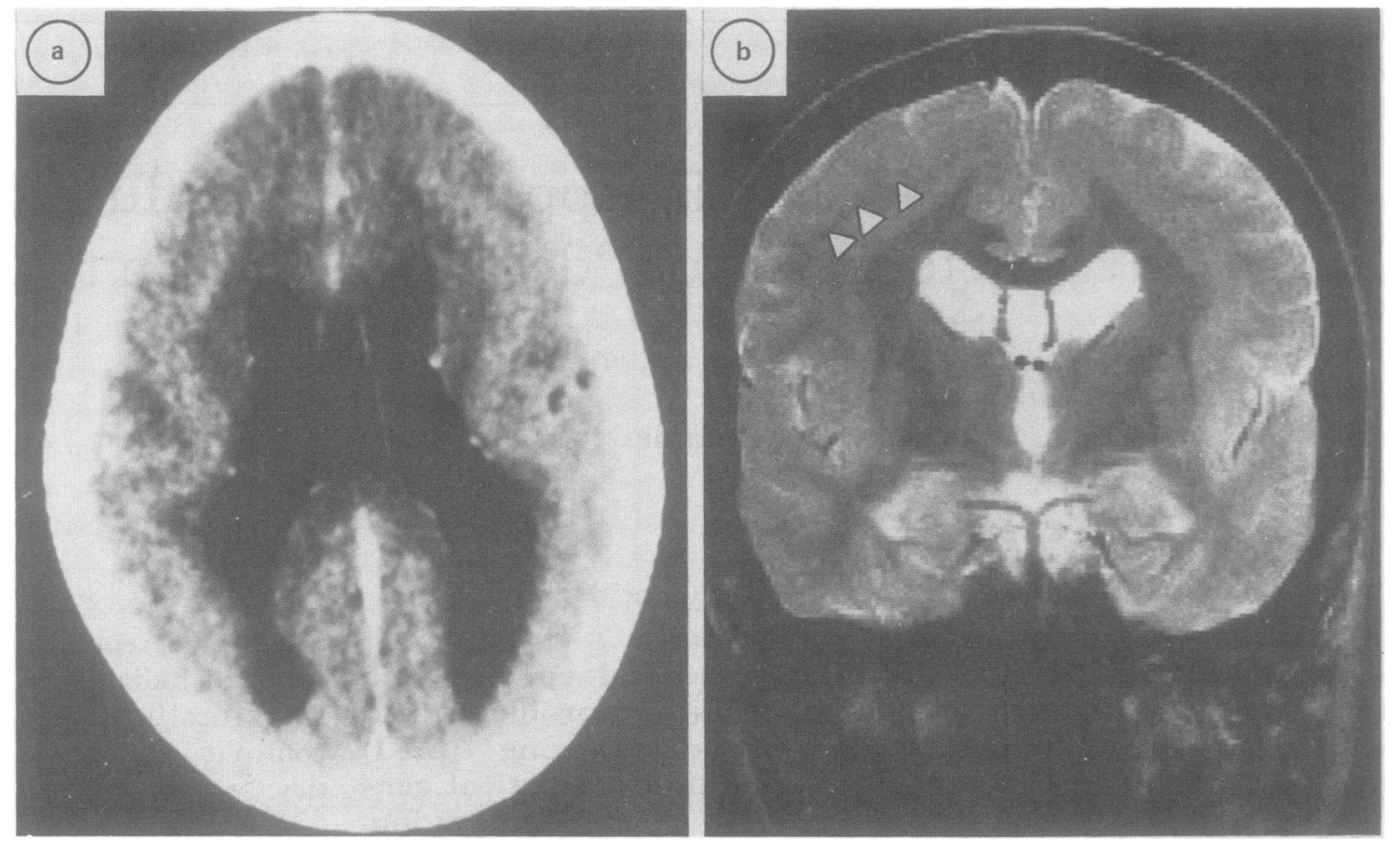

$M R I$ (fig 1)

T2 weighted coronal images showed slightly dilated lateral ventricles. The cerebral parenchyma appeared as a four-layered structure.

There was an internal layer of white matter with an abnormally smooth junction with a thick layer of tissue with the same intensity of signal as grey matter. This was surrounded by a thin layer of white matter which had essentially normal interdigitations with the overlying cortex. The cortical gyri were of slightly increased thickness but had a normal convolutional pattern. The abnormal grey layer extended from the frontal to the parietal region. This layer was also clearly seen on $T 1$ weighted sagittal images.

Case 2 This was a girl aged 11 years and six months referred because of the onset of seizures and an abnormal CT scan which suggested a leukodystrophy.

She was the second child of consanguineous parents. The pregnancy was normal and she was born at term without problems, with a birth weight of $3.5 \mathrm{kgs}$ and occipito-frontal circumference of $35 \mathrm{cms}$. There was no family history of note. Early development was normal and she was attending a normal school in a normal class for her age. She was described as an average pupil rather shy and reserved, a bit clumsy and a bit immature for her age. At the age of 11 years and four months she started to have seizures. These were of two types: sudden pallor and facial grimace with loss of consciousness and tonic seizures, lasting for a few seconds, when her arms and trunk became stiff and she fell backwards, lasting for a few seconds. She had several seizures in a few days and then started treatment with sodium valproate after which she was free from fits. An EEG recorded before treatment showed a slow background with periodic slow wave discharges. A seizure was recorded, described clinically as an absence showing a generalised symmetrical onset of high amplitude sharp wave activity followed by spike and wave discharge at 3 per second at onset but slowing towards the end of the seizure.

\section{CT Scan}

This showed slightly dilated lateral ventricles with a hypodense surrounding white matter. The white-grey junction appeared abnormally straight but was not well visualised. MRI (fig 2) axial $T 1$ weighted images showed a normal periventricular white matter signal. This white matter layer was surrounded by a thick grey matter layer extending from the frontal to the parietal region. The white-grey junction was abnormally smooth. This deep grey layer was surrounded by a thin layer of white matter which had a normal appearing junction with the over-lying cortex which was of normal appearance and thickness. Sagittal images revealed this abnormal layer of grey extending from the frontal to the occipital region. The cortical convolutional pattern and gyral thickness appeared normal in all images.

\section{Discussion}

These two patients had an unusual MRI appearance of a diffuse layer of grey matter underlying a normal looking cortex and separated from it by an apparently normal layer of white matter. Pathological confirmation of the nature of this abnormality is clearly not possible in these patients. The MRI appearances, however, suggest that this is a diffuse disorder of neuronal migration resulting in a heterotopic grey cell layer extending from the frontal to the occipital region. This appearance is different from that seen in the usual type of diffuse disorders of neuronal migration which result in a gross abnormality of the cortex, that is, pachygyria/agyria or lissencephaly. ${ }^{12}$ 
Figure 2 Case 2: (a) $T_{1}$ weighted MRI, axial cut. (b) $T$, weighted MRI, sagittal cut. A thin layer of high signal

(arrowheads) occupies the normal position of $U$ -

fibres. Underneath, a thick layer giving a signal identical to that of grey matter is visible.

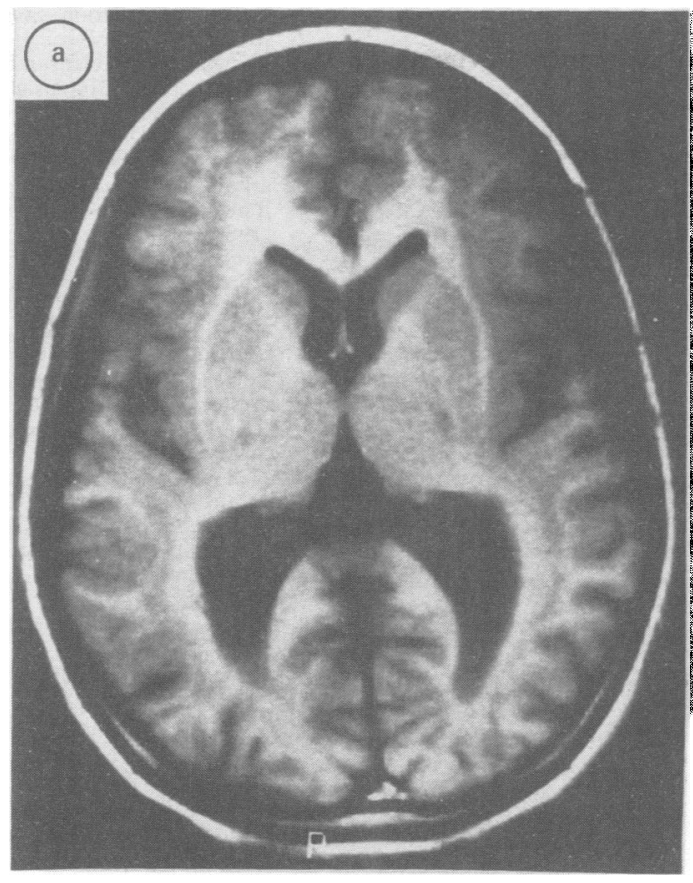

Barkovich et al have recently described five cases of "band heterotopia" whose MR images were identical to the cases we describe. ${ }^{5}$ Three of these five patients had an abnormal convolutional pattern in the overlying cortex. These appearances are also similar to the two cases described recently by Marchal et al in which a diffusely thick cerebral cortex with poor gyration and poor white-grey differentiation was shown in patients with intractable epilepsy and mental retardation. Brain biopsy in one of these cases revealed poor demarcation of cortical layers 5 and 6 with heterotopic neuronal masses in superficial white matter. This generalised cortical dysplasia may represent a mild form of lissencephaly.

Our two cases differ from these in that there was a virtually normal convolutional pattern of the superficial cortex clearly shown on MR images, and the heterotopias were more clearly defined as a diffuse subcortical grey layer.

This appearance is likewise different from the usual radiological appearance of neuronal heterotopias which show as discreet nodular grey cell masses or irregularities of the ventricular wall.

Allowing for the lack of neuropathological verification we feel that this MRI appearance may represent what has been called laminar heterotopia by the neuropathologists. ${ }^{78}$ In laminar heterotopia there are bilateral symmetrical layers of grey matter in the centrum semiovale, situated between the cortex and the ventricular wall and separated from both by well developed layers of white matter. ${ }^{79}$ The overlying cortex may be normal or may be an abnormal four layered pachygyric cortex. ${ }^{8}$

Although it is felt that laminar heterotopia, pachygyria and agyria are different degrees of the same type of lesion, distinguishing between them is necessary because the structure of the overlying cortex in laminar heterotopia may be normal. ${ }^{7}$ In both these cases the CT scan appearances were misleading, showing a

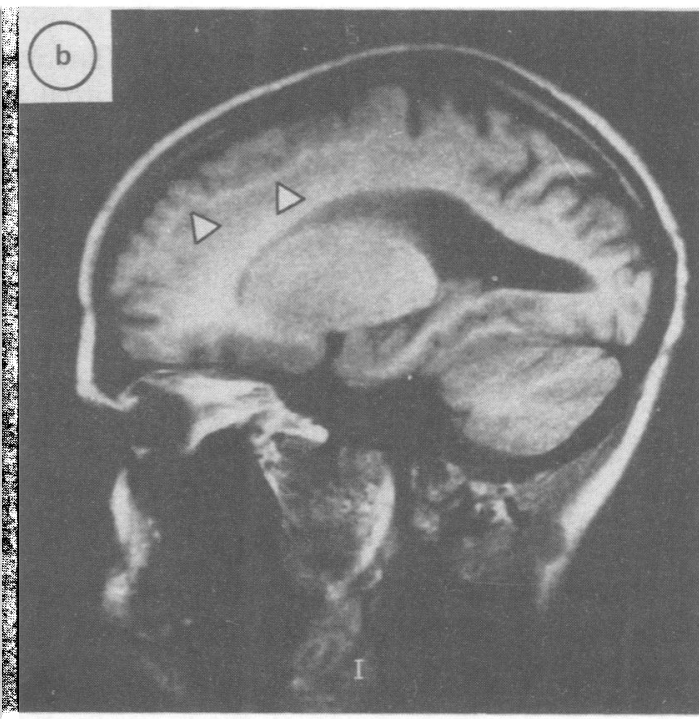

hypodense white matter. In the second patient this resulted in a provisional diagnosis of a leukodystrophy. That these patients had a malformation was not clear until MRI images were seen.

The clinical presentation of both these children was similar. They both presented with generalised seizures that responded well to medication. In addition they both had mild learning and behaviour problems but were otherwise regarded as normal children. Considering the degree of malformation present in both these children it is somewhat surprising that they are functioning so well and have so few clinical problems. The patients described by Barkovich et al, ${ }^{5}$ were more severely affected with intractable epilepsy and severe developmental delay.

Diffuse disorders of neuronal migration such as pachygyria/agyria are usually associated with severe mental retardation, intractable seizures, and early death. ${ }^{1}$ The clinical consequences of the malformation in the cases described by Marchal et al $t^{6}$ were more severe than our cases. Both cases had intractable epilepsy and mental retardation. They had, however, survived to the age of nine and 16 years. Some of the cases of laminar heterotopia described by Weist and Hallervorden ${ }^{9}$ and Friede $^{7}$ were mildly retarded adults with epilepsy, some of whom were independent in daily living.

The EEG appearances in our cases were not typical of those seen in pachygyria/agyria. ${ }^{10}$ In these disorders the EEG characteristically shows moderate to high amplitude fast activity. In our patients the predominant EEG background was slow with occasional generalised paroxysms. This was associated with generalised myoclonic or tonic seizures that responded well to sodium valproate.

These cases illustrate that it is possible to have a diffuse malformation with relatively minor clinical consequences. The reasons for this are not clear.

The cases we report are most likely to be an example of a mild form of a generalised disorder of neuronal migration. The cases recently 
described by Barkovich et $a l^{f}$ and Marchal et al have many similarities to ours and add weight to the concept that generalised disorders of neuronal migration have a spectrum of severity with mild cases such as ours at one end and pachygyria/agyria at the other. These cases again emphasise the value of MR imaging in diagnosing disorders of neuronal migration.

1 Barth PG. Disorders of neuronal migration. Can J Neurol Sci 1987;14:1-16.

2 Barkovich AJ, Chuang SH, Normal D. MR of neuronal migration anomalies. Am J Neuroradiol 1987;8:1009-17.

3 Kuzniecky $R$, Anderman F, Tampieri D, et al. Bilatera Central Macrogyria: epilepsy, pseudobulbar palsy and mental retardation-a recognisable neuronal migration disorder. Ann Neurol 1989;25:547-54.

4 Andermann F, Melanson D, Olivier A, et al. Epilepsy due to focal cortical dysplasia with macrogyria and the forme fruste of tuberous sclerosis. A study of 15 patients. In Wolff P, Dam M, Janz D, et al, eds. Advances in epileptology. New York: Raven Press, 1987; 16:35-8.

5 Barkovich AJ, Jackson DE, Boyer RS. Band heterotopias: a newly recognised neuronal migration anomaly. Radiology 1989;171:455-8.

6 Marchal G, Andermann F, Tampieri D, et al. Generalised cortical dysplasia manifested by a diffusely thick cerebral cortex. Arch Neurol 1989;46:430-4.

7 Friede RL. Developmental neuropathology. Berlin Springer-Verlag, 1989:33-5.

$8 \mathrm{Jacob} \mathrm{H}$. Genetisch verschiediene Gruppen entwicklungsgestorter Gehirne. $Z$ Ges Neurol Psychiat 1936;155:1-39.

9 Weist WD, Hallervorden J. Migrations Hemmungen im gross und Kleinhirn. Disch Z Nervenheilk 1958;178: gross 38 .

10 Gastaut H, Pinsard N, Raybaud CH, et al. Lissencephaly (Agyria-Pachygyria). Clinical Findings and Serial EEG studies. Dev Med Child Neurol 1987;29:167-80. 